\title{
Identificación de los estilos de aprendizaje de los estudiantes del curso de Física General
}

\author{
Identifying learning styles of students in the course of General Physics
}

${ }^{1}$ Julio Cesar Guevara Injoque

\begin{abstract}
Resumen
El propósito de esta investigación fue identificar los estilos de aprendizaje de los estudiantes en el curso de Física General de la Universidad Nacional Agraria la Molina sobre la base del modelo de estilos de aprendizaje desarrollado por Felder y Silverman. Se utilizó un diseño no experimental descriptivo, transversal. Participaron 363 estudiantes matriculados en el curso de Física General de los cuales 208 (57,30\%) fueron mujeres y 155 (42,70\%) fueron hombres. Para determinar los estilos de aprendizaje de los estudiantes se utilizó el Cuestionario de Estilos de Aprendizaje diseñado por Felder y Soloman. El estudio mostró que los estudiantes mostraron estilos de aprendizaje equilibrados en los estilos de aprendizaje activo, sensitivo y secuencial y moderado para el estilo visual. En cuanto al género, se encontró que las mujeres tuvieron estilo de aprendizaje activo, sensitivo y secuencial con intensidad equilibrada. Mientras que los hombres tuvieron estilos de aprendizaje reflexivo, sensitivo y secuencial con intensidad equilibrada. Ambos manifestaron una intensidad moderada para el estilo visual. Se encontró diferencias significativas entre el sexo y el estilo de aprendizaje de la dimensión comprensión Finalmente, los estudiantes de las especialidades de Biología, Ambiental, Forestal y Alimentaria tuvieron estilos de aprendizaje: activo, sensorial, visual y secuencial. Mientras que los estudiantes de las especialidades de Agronomía, Meteorología, Economía, Estadística, Gestión, Agrícola, Zootecnia y Pesquería mostraron estilos de aprendizaje reflexivo, sensorial, visual y secuencial. No se encontró diferencias significativas entre las especialidades y los estilos de aprendizaje. Por tanto, mediante el Cuestionario de Estilos de Aprendizaje de Felder y Soloman los estilos de aprendizaje de los estudiantes del curso de Fisca General pueden ser identificados y ser usados para apoyar a los estudiantes considerando sus estilos individuales de aprendizaje.
\end{abstract}

Palabras claves: aprendizaje, estilos, estilos de aprendizaje, física, género.

\begin{abstract}
The purpose of this research was to identify the learning styles of the students in the General Physics course of the National Agrarian University La Molina based on the Felder - Silverman model of learning styles. A descriptive, non-experimental cross-sectional design was used. Participated 363 students enrolled in the General Physics course of which 208 (57.30\%) were women and 155 (42.70\%) were men. To determine the learning styles of the students, the Felder and Soloman's Index of Learning Style was used. The study showed that students showed balanced learning styles in active, sensitive and sequential learning styles and moderate to visual style. As for gender, women were found to have active, sensitive and sequential learning style with balanced intensity. While men had reflexive, sensitive and sequential learning styles with balanced intensity. Both manifested a moderate intensity for the visual style. Finally, the studens of Biology, Environmental, Forestry and Food Science had learning styles: active, sensorial, visual and sequential. While the students of Agronomy, Meteorology, Economics, Statistics, Management, Agricultural, Animal Science and Fishery showed reflexive, sensorial, visual and sequential learning styles. No significant differences were found between careers of students and learning styles. Thus, through the Felder and Soloman's Index of Learning Styles, the learning styles of students of the Fisca General course can be identified and used to support students by considering their individual learning styles.
\end{abstract}

Key words: attitude, styles, learning styles, physics, gender.

\section{Introducción}

Cada estudiante en el aula procesa la información de una manera única, determinando que un estudiante se distinga de los demás. Las personas aprenden de manera diferente, y los psicólogos han intentado explicar estos diferentes modos de aprender de los estudiantes categorizando en estilos de aprendizaje (Aguilera y Ortiz, 2009). Indagar cuales son los estilos de aprendizaje de los estudiantes, permite a los docentes identificar las estrategias para facilitar y reforzar el aprendizaje de los estudiantes. Felder y Henriques (1995) consideran que el estilo de aprendizaje es la manera en la que una persona 
adquiere, conserva y recupera la información. Así, las personas aprenden de diversas formas: oyendo o viendo; reflexionando o actuando; experimentando o modelando; por pasos o por saltos. Felder y Silverman (1988) proponen que los estilos de aprendizaje se agrupan en cuatro dimensiones: procesamiento de la información, percepción de la información, representación de la información y comprensión de la información y cada dimensión está formada por dos estilos de aprendizaje opuestos.

La dimensión de Procesamiento está formada por los estilos de aprendizaje activo y reflexivo, de este modo los estudiantes con estilo de aprendizaje activo aprenden mejor trabajando activamente con el material de aprendizaje, y probando cosas. En cambio, los estudiantes con estilo de aprendizaje reflexivo prefieren pensar y reflexionar sobre el material. La dimensión de Percepción está integrada por los estilos de aprendizaje sensorial e intuitivo, de esta manera los estudiantes con un estilo de aprendizaje sensorial prefieren aprender hechos y con materiales de aprendizaje concreto. En contraste, los estudiantes intuitivos prefieren materiales de aprendizaje abstracto, como teorías y sus significados profundos, son innovadores y creativos.

La dimensión de Representación está constituida por los estilos de aprendizaje visual y verbal. Los estudiantes con estilo visuales aprenden mejor con imágenes, videos o demostraciones en aula. Por otra parte, los estudiantes con estilo de aprendizaje verbal prefieren las explicaciones escritas y orales. La dimensión Comprensión está conformada por los estilos de aprendizaje secuencial y global. Los estudiantes con estilo de aprendizaje secuencial aprenden en pequeños pasos incrementales y por lo tanto tienen un progreso de aprendizaje lineal y están más interesados en los detalles. En cambio, los estudiantes con estilo de aprendizaje global utilizan el pensamiento holístico y aprenden en grandes saltos y tienden a estar más interesados en las descripciones. Las investigaciones han mostrado que los estudiantes de ciencias e ingeniería tienden a tener preferencia hacia estilos de aprendizaje activo, sensorial, visual y secuencial (Checa, 2011; Solís y Arcudia, 2010 y Troiano, Breitma y Gete-Alonso, 2004).

El propósito de esta investigación es identificar los estilos de aprendizaje de los estudiantes que están en el curso de Física General y describir sus estilos de aprendizaje conforme al sexo y especialidad de los estudiantes. La información será útil no sólo en la determinación de las estrategias de enseñanza apropiados para que coincida con los estilos de aprendizaje de los estudiantes, sino también en la mejora del rendimiento académico de los estudiantes del curso.

\section{Métodos y materiales Participantes}

Los participantes fueron 363 estudiantes regulares de las diferentes especialidades de la Universidad que estaban matriculados en el curso de Física General. Esta muestra de conveniencia incluyó a 208 (57,30\%) mujeres y 155 $(42,70 \%)$ hombres, con una edad comprendida entre 18 y 26 años $(M=19,36 ; D E=1,71)$. Se excluyeron los estudiantes que faltaron el día de la aplicación del cuestionario.

\section{Instrumento}

Para la recolección de los datos se usó el Cuestionario de Estilos de Aprendizaje de Felder y Soloman (Felder y Soloman; 2004). El instrumento fue adaptado al español por Rodríguez (2002) y está conformado por 44 ítems y diseñado a partir de cuatro dimensiones -Percepción, Procesamiento, Representación y Comprensión- donde cada dimensión está vinculada con dos estilos de aprendizaje opuestos.

Con respecto al puntaje que se obtiene en el instrumento cada pregunta es de un punto. De esta manera, para cada dimensión hay un total de 11 puntos. Este puntaje se interpreta de acuerdo a tres niveles de intensidad: equilibrado, moderado y fuerte. Así, unas puntuaciones de 1 a 3 puntos representan una intensidad equilibrada indicando un balance entre los dos estilos de aprendizaje de esta dimensión. Puntajes de 5 a 7 puntos, muestran una intensidad moderada, esto es, hay una leve preferencia hacia uno de los estilos de aprendizaje de esta dimensión. Y puntuaciones de 9 a 11 puntos, señalan una fuerte preferencia hacia uno de los estilos de aprendizaje de esta dimensión.

Para cada dimensión se tienen once preguntas de respuesta forzada a dos opciones, A y $\mathrm{B}$, donde la opción A corresponde a los estilos de aprendizaje activo, sensorial, visual y secuencial. La opción B se refiere a los estilos de aprendizaje reflexivo, intuitivo, verbal y global. Diversos estudios sobre estilos de aprendizaje han aplicado el Cuestionario de Estilos de Aprendizaje de Felder y Soloman en el contexto Latinoamericano así como fuera de él. En el ámbito Latinoamericano distintos estudios han mostrado que el instrumento es fiable y valido para identificar los estilos de aprendizaje de los estudiantes de ciencias e ingeniería (Ocampo, Guzmán, Camarena y De Luna; 2014, Guanipa y Mogollón, 2006; Troiano, Breitma y Gete-Alonso, 2004).

Con respecto a la validez y confiabilidad del instrumento, Gamero (2012), determinó la validez de contenido, validez de constructo y confiabilidad del Cuestionario de Estilos d Aprendizaje de Felder y Soloman. La validez de contenido se realizó a través de juicio de expertos para lo cual se entrevistó a cinco doctores en educación dando como resultado un coeficiente de 0,89 . La validez de constructo se hizo a través de intra-prueba, para ello se estructuró una matriz de correlaciones de Pearson entre los ítems, y las dimensiones dando un valor promedio para el instrumento de 0,58 . Además, la confiabilidad del instrumento fue medida a través del alfa de Cronbach el cual fue de 0,62. Por otro lado, Ocampo et al. (2014), midieron la validez de constructo mediante el análisis factorial y confirmó que el Cuestionario de Estilos d Aprendizaje de Felder y Soloman tiene cuatro 
dimensiones. La confiabilidad medida a través del alfa de Cronbach fue de 0,62. Para la presente investigación la confiabilidad medida a través del alfa de Cronbach fue de 0,60 .

\section{Diseño}

El enfoque utilizado en esta investigación fue el cuantitativo con un diseño no experimental transversal del tipo descriptivo (Hernández, Fernández y Baptista, 2010)

\section{Procedimiento}

Los datos fueron recolectados en cada grupo del curso de Física durante el segundo semestre del 2014, en el primer día de clase y en coordinación con los profesores del curso. Los estudiantes que estaban presentes en el aula fueron informados que la investigación tenía una naturaleza académica y era anónima siendo voluntario participar en el estudio. Luego, se procedió a distribuir el Cuestionario de Estilos de Aprendizaje entre los estudiantes donde primero contestaron sobre su edad, sexo y especialidad y posteriormente respondieron a las preguntas del cuestionario. Al completar el cuestionario los estudiantes procedieron a devolverla al profesor y el tiempo que tomaron en responder los estudiantes al instrumento fue de 20 minutos aproximadamente.

\section{Resultados}

\section{Estilos de aprendizaje de los estudiantes}

La tabla 1, muestra que los estudiantes tuvieron un estilo de aprendizaje activo, sensorial, visual y secuencial. Por otro lado, los estudiantes con estilo de aprendizaje activo $(50,69 \%)$ no fueron significativamente diferente que los estudiantes con estilo de aprendizaje reflexivo (49,31\%). Además, los estudiantes con estilos de aprendizaje sensorial $(69,79 \%)$ tuvieron diferencias significativas con los estudiantes con estilos de aprendizaje intuitivo (30,03\%). Asimismo, el estilo de aprendizaje visual $(85,40 \%)$ de los estudiantes fue significativamente diferente que el de los estudiantes con estilos de aprendizaje verbal (14,60\%). También, hubo diferencia significativa entre los estudiantes con estilo de aprendizaje secuencial $(74,10 \%)$ y los estudiantes con estilo de aprendizaje global $(25,90 \%)$.

Tabla 1. Estilo de aprendizaje de los estudiantes del curso de Física General

\begin{tabular}{llcc}
\hline \multicolumn{1}{c}{ Dimensión } & \multicolumn{1}{c}{ Estilo } & $\mathrm{n}$ & $\%$ \\
\hline \multirow{2}{*}{ Procesamiento } & Activo & 184 & 0,38 \\
& Reflexivo & 179 & \\
\multirow{2}{*}{ Percepción } & Sensorial & 254 & $7,08 * * *$ \\
& Intuitivo & 109 & \\
Representación & Visual & 310 & $10,84 * * *$ \\
& Verbal & 53 & \\
Compresión & Secuencial & 269 & \multirow{2}{*}{$8,24 * * *$} \\
& Global & 94 & \\
\hline
\end{tabular}

$\mathrm{N}=363, * * * \mathrm{p}<0,001$
La mayoría de los estudiantes mostraron estilos de aprendizaje equilibrados, con excepción del estilo de aprendizaje visual que tuvo una intensidad moderada (tabla 2).

Tabla 2. Intensidad de los estilos de aprendizaje de los estudiantes del curso de Física General

\begin{tabular}{lcccccc}
\hline Estilos & \multicolumn{2}{c}{ Equilibrado } & \multicolumn{2}{c}{ Moderado } & \multicolumn{2}{c}{ Fuerte } \\
\cline { 2 - 7 } & $\mathrm{n}$ & $\%$ & $\mathrm{n}$ & $\%$ & $\mathrm{n}$ & $\%$ \\
\hline Activo & 127 & 69,02 & 50 & 27,17 & 7 & 3,80 \\
Reflexivo & 117 & 65,36 & 58 & 32,40 & 4 & 2,23 \\
Sensorial & 155 & 61,02 & 87 & 34,25 & 12 & 4,72 \\
Intuitivo & 93 & 85,32 & 14 & 12,84 & 2 & 1,83 \\
Visual & 96 & 30,97 & 148 & 47,74 & 66 & 21,29 \\
Verbal & 43 & 81,13 & 9 & 16,98 & 1 & 1,89 \\
Secuencial & 144 & 53,53 & 97 & 36,06 & 28 & 10,41 \\
Global & 79 & 84,04 & 13 & 13,83 & 2 & 2,13 \\
\hline N=363 & & & & & &
\end{tabular}

Estilos de aprendizaje y sexo de los estudiantes

La tabla 3, muestra que las mujeres tuvieron un estilo de aprendizaje activo, sensorial, visual y activo además los hombres tuvieron estilos de aprendizaje reflexivo, sensorial, visual y secuencial. Asimismo, no se encontró diferencias significativas entre mujeres y hombres con los estilos de aprendizaje activo - reflexivo , sensorial - intuitivo y visual - verbal. Sin embargo, si hubo diferencias significativas entre mujeres y hombres con los estilos de aprendizaje secuencial - global .

Tabla 3. Estilos de aprendizaje de los estudiantes por sexo

\begin{tabular}{lcccc}
\hline \multirow{2}{*}{ Estilos } & \multicolumn{2}{c}{ Mujer } & \multicolumn{2}{c}{ Hombre } \\
& $\mathrm{n}$ & $\%$ & $\mathrm{n}$ & $\%$ \\
\hline Activo & 109 & 30,03 & 75 & 20,66 \\
Reflexivo & 99 & 27,27 & 80 & 22,04 \\
Sensorial & 148 & 40,77 & 106 & 29,20 \\
Intuitivo & 60 & 16,53 & 49 & 13,50 \\
Visual & 180 & 49,59 & 130 & 35,81 \\
Verbal & 28 & 7,71 & 25 & 6,89 \\
Secuencial & 165 & 45,45 & 104 & 28,65 \\
Global & 43 & 11,85 & 51 & 14,05 \\
\hline
\end{tabular}

$\mathrm{N}=363$

La figura 1, muestra que la mayoría de los estilos de aprendizaje de las estudiantes mujeres tuvieron una intensidad equilibrada a excepción del estilo visual que fue de intensidad moderada. Igualmente, la mayoría de los estudiantes hombres tuvieron estilos de aprendizaje con una intensidad equilibrada con excepción del estilo de aprendizaje visual que tuvo una intensidad moderada. 


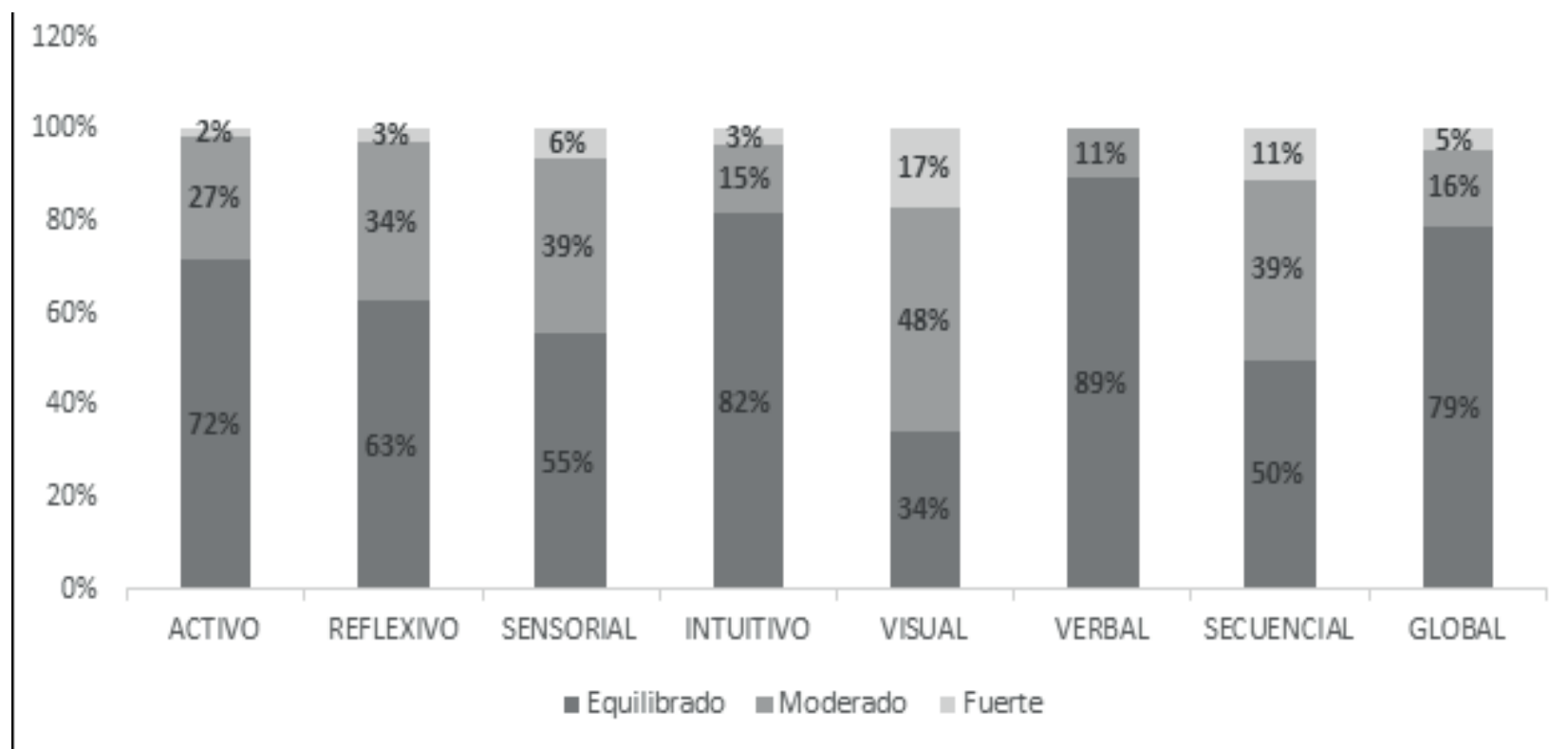

Figura 1. Intensidad de los estilos de aprendizaje para las estudiantes mujeres

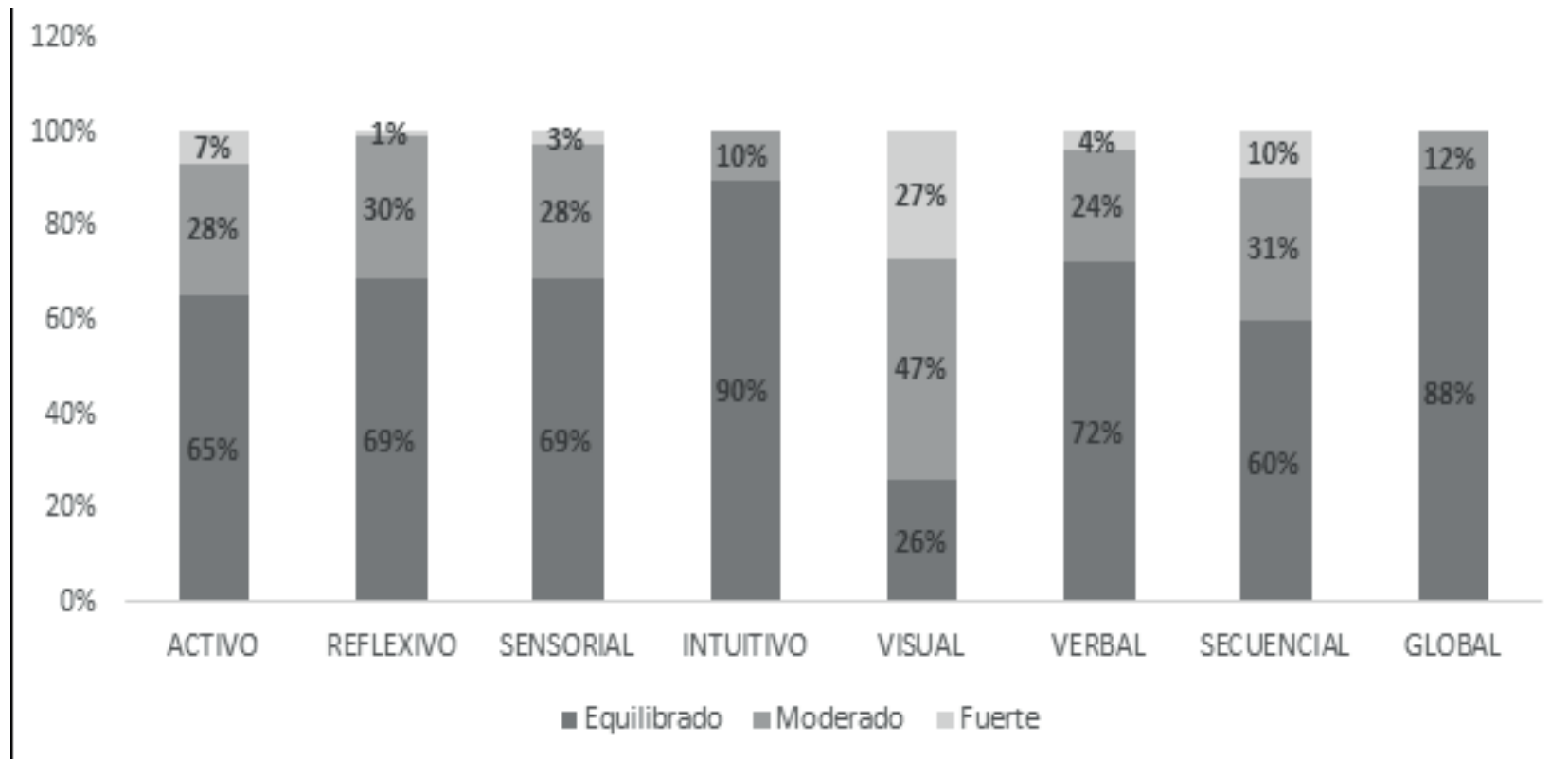

Figura 2. Intensidad de los estilos de aprendizaje para los estudiantes hombres

\section{Estilos de aprendizaje y especialidad de los estudiantes} La tabla 4, muestra que los estudiantes de Biología, Ambiental, Forestal y Alimentaria tuvieron estilos de aprendizaje: activo, sensorial, visual y secuencial. Mientras que los estudiantes de Agronomía, Meteorología, Economía, Estadística, Gestión, Agrícola, Zootecnia y Pesquería mostraron estilos de aprendizaje:reflexivo, sensorial, visual y secuencial. La mayoría de las especialidades mostraron un estilo de aprendizaje visual con la excepción de la especialidad de Ambiental. Por otra parte, no se encontraron diferencias significativas entre las especialidades de los estudiantes y los estilos de aprendizaje activo - reflexivo $(\chi 2(11,363)=15,35) ; p$ $=0,17)$, sensorial - intuitivo $(\chi 2(11,363)=9,79) ; p=0,55)$, visual $\left.-\operatorname{verbal}\left(\chi^{2}(11,363)=8,96\right) ; p=0,63\right)$ y secuencial $\left.-\operatorname{global}\left(\chi^{2}(11,363)=11,14\right) ; \mathrm{p}=0,43\right)$. 
Tabla 4. Estilos de aprendizaje de los estudiantes por especialidad

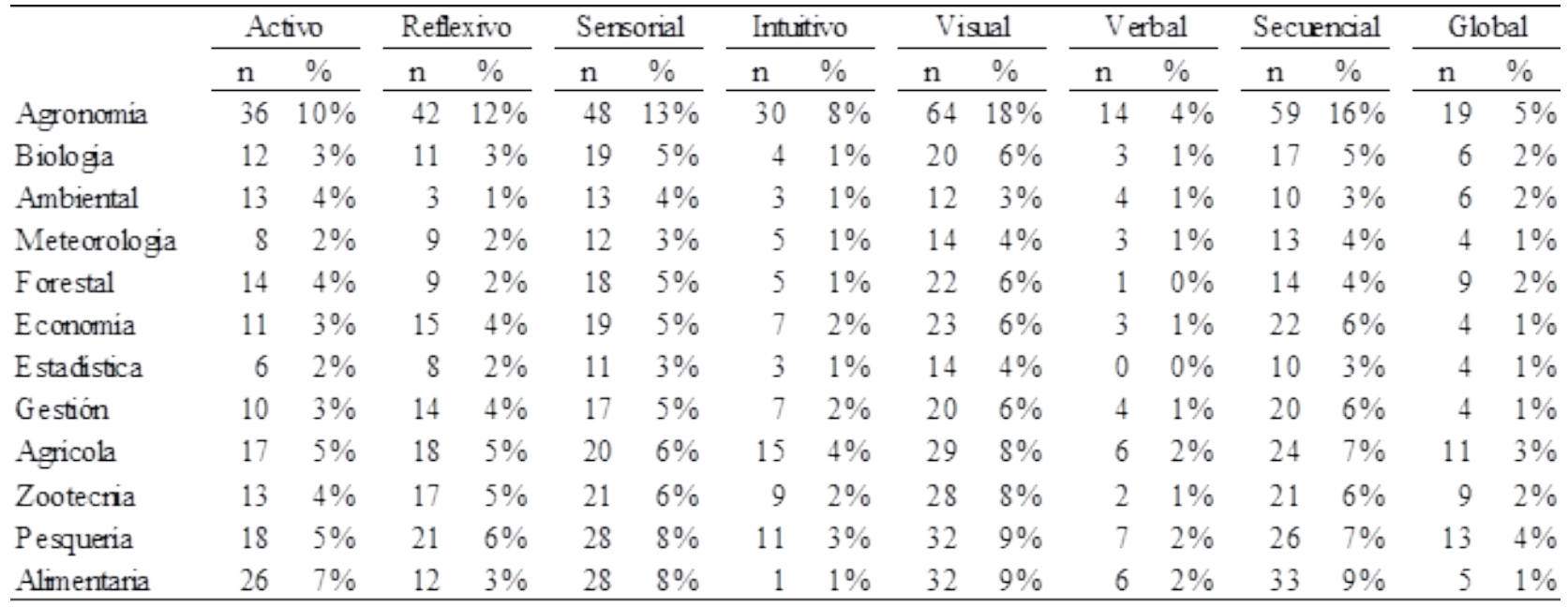

$\mathrm{N}=363$

\section{Discusión}

El propósito de esta investigación fue determinar los estilos de aprendizaje de los estudiantes del curso de Física General durante el segundo semestre del año 2014; a través de la aplicación del Cuestionario de Estilos de Aprendizaje de Felder y Soloman (Felder y Soloman; 2004).

En relación a los estilos de aprendizaje de los estudiantes del curso de Fisca General estos mostraron tener estilos de aprendizaje visual, secuencial, sensorial y activo. Esto indica que los estudiantes de la muestra del curso de Física tienen una preferencia hacia la presentación de materiales visuales recuerdan mejor lo que ven y también les agrada aprender en pasos secuenciados y lógicamente ordenados. También, tienden a ser prácticos orientados hacia los hechos y procedimientos además les gusta explicar lo que quieren comprender. Estos resultados obtenidos coinciden con los resultados reportados por Ventura, Moscoloni y Gagliardi (2012) y Soto y otros (2008) donde encontraron que los estilos de aprendizaje de los estudiantes de ingeniería tienden a ser activos, sensoriales, visuales y secuenciales. Igualmente, se encontró que hubo diferencias entre los estilos de aprendizaje en las dimensiones de percepción, representación y comprensión; pero no hubo diferencias en la dimensión de procesamiento.

Además, tanto estudiantes mujeres como hombres tuvieron un estilo de aprendizaje mayoritariamente visual con una intensidad moderada. También, se encontró que en la dimensión procesamiento las mujeres tuvieron un estilo de aprendizaje activo, es decir gustan de discutir, aprenden mejor trabajando en grupo. Mientras, que los hombres tuvieron un estilo de aprendizaje reflexivo, esto es aprenden mejor si medita la información que se muestra. Para la dimensión de percepción se encontró que mujeres y hombres tuvieron un estilo de aprendizaje sensorial esto es gustan de aprender de manera ordenada y lógica. Asimismo, para la dimensión de representación tanto mujeres como hombres fueron visuales es decir a ambos les gusta recibir la información a través de figuras y diagramas. Del mismo modo para la dimensión de comprensión mujeres y hombres tuvieron un estilo de aprendizaje secuencial o sea tienden a procesar la información por partes para luego entender toda la información. Por otra parte, no se encontró diferencias significativas entre el sexo y los estilos de aprendizaje de las dimensiones de procesamiento, percepción y representación pero se halló que existe una diferencia significativa en la dimensión de comprensión. Estos resultados coinciden para las dimensiones de percepción y representación pero discrepan con las dimensiones de procesamiento y comprensión obtenidos por Ocampo et al. (2014).

Con relación a las especialidades se encontró que los estudiantes de Biología, Ambiental, Forestal y Alimentaria aprenden mejor a través de la participación en clases, prefieren hechos; les gusta recibir la información a través de figuras o diagramas y aprender por pasos lógicos. En cambio los estudiantes de Agronomía, Meteorología, Economía, Estadística, Gestión, Agrícola, Zootecnia y Pesquería aprenden mejor pensando sobre los temas en clase, prefieren hechos; les gusta recibir la información a través de figuras o diagramas y aprender por pasos lógicos. Asimismo, no se encontraron diferencias significativas entre las especialidades de los estudiantes las dimensiones de procesamiento, percepción, representación y compresión.

\section{Conclusión}

Los resultados mostraron que en general los estudiantes aprenden mejor viendo imágenes, diagramas o demostraciones. Además, gustan de resolver problemas de manera lógica y secuencial y también son observadores y recogen hechos a través de la experimentación. Asimismo les gusta aprender a través de la discusión o constatar la información de alguna manera.

Por otro lado, los estudiantes mostraron estilos de aprendizaje secuencial, sensitivo y activo y con una 
intensidad equilibrada. Esto permite que los estudiantes se sientan cómodos en cuanto a las estrategias de enseñanza de los profesores. Sin embargo el estilo de aprendizaje visual mostro una intensidad moderada es decir, los estudiantes prefieren que el material de enseñanza se presentado a través de imágenes para aprender de manera más óptima.

A su vez, los estilos de aprendizaje que expresaron los estudiantes, mujeres y hombres, son similares para los estilos de aprendizaje visual, secuencial, sensitivo. Pero las mujeres mostraron un estilo de aprendizaje activo mientras que los hombres mostraron un estilo de aprendizaje reflexivo.

Así pues, el conocer los estilos de aprendizaje de los estudiantespermite a los profesores proponer estrategias de aprendizaje que permitan favorecer el aprendizaje de los estudiantes en los diversos temas impartidos para las diferentes especialidades de la universidad.

\section{Referencias}

Aguilera, E. y Ortiz, E. 2009. Las investigaciones sobre los estilos de aprendizaje y sus modelos explicativos. Revista Estilos de Aprendizaje, 4,4.

Checa, P. 2011. La innovación metodológica en la enseñanza de la programación, una aproximación pedagógica al aprendizaje activo en la asignatura fundamentos de programación. INTERFASES, 4, 2011, 67-87

Felder, R. y Henriques, E. 1995. Learning and teaching styles in foreign and second language education. Foreign Language Annals, 28(1), 21-31.

Felder, M. y Silverman, L. 1988. Learning and teaching styles in engineering education. Journal of Engineering Education, 78(7), 674-681.

Felder, M. y Soloman, B. 2004. Soloman. Index of learning styles. Recuperado de http://www4.ncsu.edu/ unity/lockers/users/f/felder/public/ILS-faq.htm

Gamero, P. 2012. Estilos de Aprendizaje y su influencia en el rendimiento académico de estadística, por los maestrandos de la escuela de post grado de la Universidad Nacional de Educación Enrique Guzmán y Valle durante el semestre académico 2011-1. (Tesis de Doctor), Universidad Nacional de Educación Enrique Guzmán y Valle, La Molina, Perú.

Guanipa, M. y Mogollón, E. 2006. Estilos de aprendizaje y estrategias cognitivas en estudiantes de ingeniería. Revista Ciencias de la Educación, 1,27.

Hernández, R.. Fernández, C. y Baptista, P. 2010. Metodología de la investigación. México: McGraw-Hill/ Interamericana Editores

Ocampo, F.; Guzmán, A.; Camarena, P. y De Luna, R. 2014. Identificación de estilos de aprendizaje en estudiantes de ingeniería. Revista Mexicana De Investigación Educativa, 19 (61).

Solís, R. y Arcudia, C. 2010. Estilos de aprendizaje de los estudiantes de ingeniería civil. Revista Educación en Ingeniería, 10, 2010.
Soto, E., Azevedo, A. y Cunha, C. 2008. Estilos de aprendizagem de Felder-Soloman dos alunos ingressantes em engenharia agronômica e engenharia florestal,

ESALQ/USP, 2008. Simpósio Internacional de Iniciação Científica e Tecnológica da USP 2008.

Troiano, H., Breitman, M. y Gete-Alonso, C. 2004. Estilos de aprendizaje que predominan entre los estudiantes universitarios. Revista de Enseñanza Universitaria, 23, 63-84.

Ventura, C.; Moscoloni, N. y Gagliardi, R. 2012. Estudio comparativo sobre los estilos de aprendizaje e estudiantes universitarios argentinos de diferentes disciplinas. Psicología Desde El Caribe, 29(2). 